\title{
Isolated Ossification Site
}

National Cancer Institute

\section{Source}

National Cancer Institute. Isolated Ossification Site. NCI Thesaurus. Code C124551.

An ossification site not localized to the main ossification area, as indicated by staining,

but is still considered to be within the margins of a normal bone precursor. 